\title{
Adhesive cementation of zirconia posts to root dentin: evaluation of the mechanical cycling effect
}

\author{
Graziela Ávila Galhano(a) \\ Renata Marques de Melo(a) \\ Carlos Augusto Pavanelli(b) \\ Paolo Baldissara(c) \\ Roberto Scotti(d) \\ Luiz Felipe Valandro(e) \\ Marco Antonio Bottino(f)
}

(a) PhD Students in Prosthodontics; (b) PhD, Associate Professor; (f) PhD, Professor - Dept. of Dental Materials and Prosthodontics, School of Dentistry of São José dos Campos, São Paulo State University, São José dos Campos, SP, Brazil.

(c) Professor and Researcher; (d)MD, DDS, Chairman and Professor - Dept. of Oral Science, Alma Mater Studiorum, University of Bologna, Italy.

(e) MSc, Assistant Professor, Dept. of Restorative Dentistry, Federal University of Santa Maria, Santa Maria, RS, Brazil.

\section{Corresponding author:}

Graziela Ávila Prado Galhano

Universidade Estadual Paulista (UNESP)

Faculdade de Odontologia

Dept. de Materiais Dentários e Prótese

Av. Francisco José Longo, 777,

São José dos Campos - SP - Brazil

CEP: 12245-000

E-mail: grazielagalhano@yahoo.com.br

\begin{abstract}
This study evaluated the effect of mechanical cycling on the bond strength of zirconia posts to root dentin. Thirty single-rooted human teeth were transversally sectioned to a length of $16 \mathrm{~mm}$. The canal preparation was performed with zirconia post system drills (CosmoPost, Ivoclar) to a depth of $12 \mathrm{~mm}$. For post cementation, the canals were treated with total-etch, 3-steps All-Bond 2 (Bisco), and the posts were cemented with Duolink dual resin cement (Bisco). Three groups were formed $(n=10)$ : G1 - control, no mechanical cycling; G2 - 20,000 mechanical cycles; G3 - 2,000,000 mechanical cycles. A 1.6-mm-thick punch induced loads of $50 \mathrm{~N}$, at a $45^{\circ}$ angle to the long axis of the specimens and at a frequency of $8 \mathrm{~Hz}$ directly on the posts. To evaluate the bond strengths, the specimens were sectioned perpendicular to the long axis of the teeth, generating 2 -mm-thick slices, approximately (5 sections per teeth), which were subjected to the push-out test in a universal testing machine at a $1 \mathrm{~mm} / \mathrm{min}$ crosshead speed. The push-out bond strength was affected by the mechanical cycling (1-way ANOVA, $\mathrm{p}=.0001)$. The results of the control group $(7.7 \pm 1.3 \mathrm{MPa})$ were statistically higher than those of G2 $(3.9 \pm 2.2 \mathrm{MPa})$ and $\mathrm{G} 3(3.3 \pm 2.3 \mathrm{MPa})$. It was concluded that the mechanical cycling damaged the bond strength of zirconia posts to root dentin.
\end{abstract}

Descriptors: Post and core technique; Ceramics; Stress, mechanical; Bond strength. 


\section{Introduction}

Ceramic restorations may need aesthetic intraradicular retainers when applied on teeth that have lost their coronary portion. Aesthetic posts are needed since traditional metallic posts inhibit light transmission, alter the restoration color and promote a rather thick, grayish line on the marginal gum. ${ }^{1-5}$

Ceramic posts were developed with a color similar to that of the dental structure, optimizing the aesthetic result of restorations due to the absorption of light transmitted through the ceramic. Zirconium posts have greater resistance when compared to aesthetics posts made from other materials. ${ }^{6}$ They also meet one important requirement of intra-radicular retainers: appropriate hardness, which should be enough to offer good retention to the restoration.

From the mechanical point of view, it is believed that a rigid post contributes to the stability of the post-restoration set. However, root fractures observed when using these posts can occur as a result of the difference in stiffness between post and the remaining root structure, resulting in stress concentration on the tooth during masticatory function. ${ }^{4,7,8}$

One factor that can improve the resistance to fracture of teeth needing restoration is adhesive cementation. ${ }^{9,10}$ The use of resin cements for post cementation makes roots less vulnerable to fracture under static loads and preserves dental structure, given that the posts may be shorter and have a smaller diameter. ${ }^{4}$ However, no studies have evaluated the bond strength of ceramic posts to root dentin after mechanical cycling, simulating a masticatory load.

Clinical evidence indicates that most of the fractures in prosthetic restorations occur after many years. These fractures are not usually related to an impact, but result from long periods of mechanical fatigue, ${ }^{11,12}$ most likely explained by the development of little cracks on tension concentration areas. ${ }^{13}$ Static load tests for evaluating failures in restorations have been replaced by mechanical cycling, providing greater accuracy in simulating the loads that act on restorative materials during clinical function. ${ }^{6}$

Considering the present knowledge gap on the resistance to fatigue of ceramic posts adhesively cemented, the aim of this study was to evaluate the effect of mechanical cycling on the push-out bond strength between root dentin and zirconium post.

\section{Material and Method}

Thirty human uniradicular teeth that were extracted for orthodontic or periodontal reasons, without caries or restoration on the cervical third, were used. The Committee of Ethics in Research, São Paulo State University (UNESP, São José dos Campos, SP, Brazil), approved the project.

The dental crown was removed from each specimen and the root length was standardized to $16 \mathrm{~mm}$. The root canals were then prepared under constant irrigation with distilled water, ${ }^{14}$ followed by a thermo-plasticized gutta-percha filling.

The middle and cervical thirds were prepared with the \#2 drill of a zirconium oxide post system (Cosmopost, Ivoclar-Vivadent, Liechtenstein). The features of the zirconia post were length of $20 \mathrm{~mm}$, conic shape and average diameter of $1.7 \mathrm{~mm}$ ).

The following procedures were followed for post cementation:

a. Post surface treatment (Cojet system, ESPE, St. Paul, MN, USA): air-abrasion with $30 \mu \mathrm{m}$ aluminum oxide modified with silica, rotating the post manually until its surface appeared matte under visual inspection (air-particle protocol: pressure $=2.8$ bars; distance $=10 \mathrm{~mm}$; perpendicular to the post surface; time $=20 \mathrm{~s}$ ). Thereafter, ESPE-Sil silane was applied and allowed to dry for $5 \mathrm{~min}$.

b. Root canal dentin treatment: a multiple-bottle, total-etch adhesive system (All Bond $2^{\circledR}$ system, Bisco, Schaumburg, IL, USA) was used as follows: 1) etching with $32 \%$ phosphoric acid for $30 \mathrm{~s}$; 2) washing with $10 \mathrm{ml}$ of water using a disposable syringe; 3 ) removal of excess water with no. 80 absorbent paper points; 4) application of a mixture of Primer A and Primer B (All Bond 2 system) and removal of excess material with a Cavi-tip brush (Svenska Dental Instrument AB, Upplands Värby, Sweden); 5) application of PreBond resin (All Bond 2 system) and removal of excess material with brush.

c. Post cementation: 1) the A and B pastes of the resin cement Duolink (Bisco, Schaumburg, IL, USA) were measured and mixed. The cement 
was applied to the post and the root canal was filled with cement with a Lentulo no. 40 spiral (Dentsply Maillefer, Ballaigues, Switzerland); 2) light-curing through the incisal surface for $40 \mathrm{~s}$ with an XL 3000 unit (3M ESPE, St Paul, MN, USA) at a light intensity of $450 \mathrm{~mW} / \mathrm{cm}^{2}$.

For mechanical cycling, the specimens were embedded in epoxy resin (285, Schaller, Florence, Italy), using a support with $16 \mathrm{~mm}$ in diameter specific for each post, with a central orifice slightly larger than the coronal diameter of the post. This allowed the specimen to be as perpendicular as possible to the ground. A $10 \mathrm{ml}$ surgical plastic syringe was sectioned in a mechanical lathe so that the surface of this section was parallel to the ground $(\varnothing=12 \mathrm{~mm} ; \mathrm{H}=40 \mathrm{~mm}$ ). Thus, when the support-specimen set was positioned in the syringe, the specimen was embedded as perpendicular as possible to the ground. Following this procedure, the epoxy resin was prepared and poured into the syringe. The support with the coupled specimen was positioned into the syringe, so that the root was embedded $3 \mathrm{~mm}$ in apical direction (Figure 1).

The specimens were placed in a metallic base at a $45^{\circ}$ angle, so that a point with $1.6 \mathrm{~mm}$ in diameter at the upper rod of the cycling machine could induce

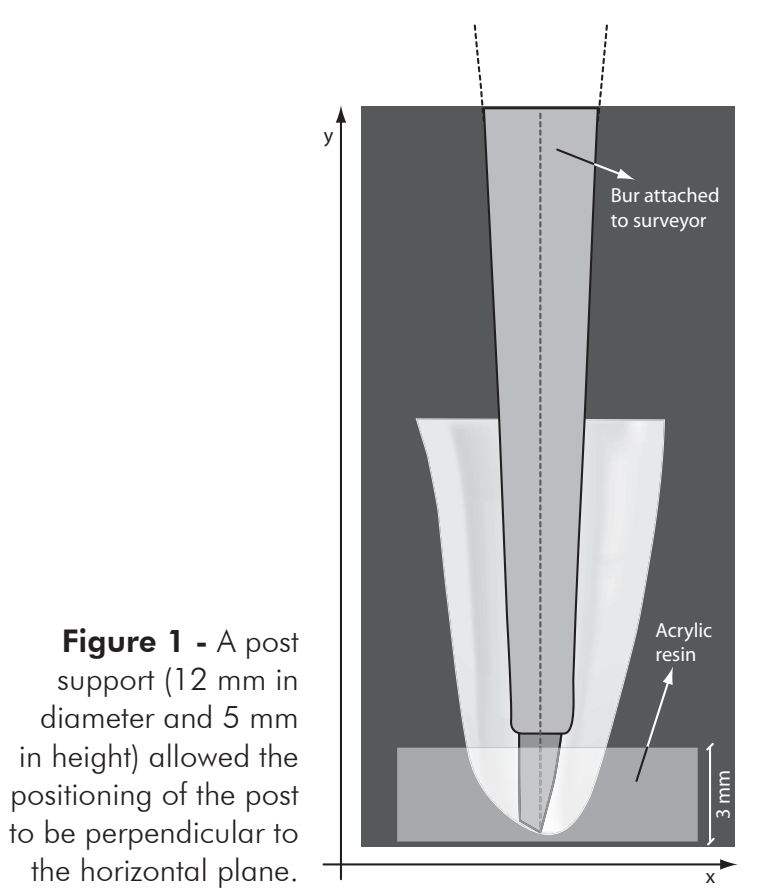

load pulses of $50 \mathrm{~N}$, at a frequency of $8 \mathrm{~Hz},{ }^{15}$ on the post surface. During cycling, the specimens were irrigated with distilled water at $37^{\circ} \mathrm{C} \pm 2^{\circ} \mathrm{C}$, regulated by a thermostat. ${ }^{12,16,17}$ The following groups $(\mathrm{n}=10)$ were composed, according to the number of cycles completed: Group 1 (G1) - no mechanical cycling; Group 2 (G2) - submitted to 20,000 mechanical cycles; Group 3 (G3) - submitted to 2,000,000 mechanical cycles.

\section{Push-out strength test and statistical analyses}

The specimens were bonded to a metallic base with cyanoacrylate adhesive gel (Super Bonder gel, Loctite-Henkel Ltda., Itapevi, SP, Brazil). The metallic base was connected to a sectioning machine and the teeth were sectioned perpendicular to their long axis with a diamond saw in 2-mm slices, under water irrigation. The first $1 \mathrm{~mm}$ section was discarded because the excess cement could lead to overestimation of the bond strength values at this segment. ${ }^{18,19}$ Overall, five segments were obtained, with 5 disc-samples per tooth (50 per group).

For push-out testing, a metallic cylinder $(0.85 \mathrm{~mm}$ in diameter) was pressed onto the post center. The test was performed in a universal testing machine (Emic DL-1000, Emic, São José dos Pinhais, PR, Brazil) at a $1 \mathrm{~mm} / \mathrm{min}$ speed. ${ }^{20}$

The push-out bond strength (S) was obtained with the formula:

$$
\begin{aligned}
& S=\text { F/A } \\
& \text { where: } \\
& \text { F: load for sample rupture }(\mathrm{kgf}) \text { and } \\
& \text { A: adhered area }\left(\mathrm{mm}^{2}\right) \text {. }
\end{aligned}
$$

To determine the adhered area, the formula to calculate the lateral area of a circular straight cone with parallel bases (Figure 2) was used. The formula is defined as:

$$
\begin{aligned}
& \mathrm{A}=\pi \times \mathrm{g} \times\left(\mathrm{R}_{1}+\mathrm{R}_{2}\right) \\
& \text { where: } \\
& \pi: 3.14, \\
& \mathrm{~g}: \text { slant height, } \\
& \mathrm{R}_{1}: \text { smaller base radius, } \\
& \mathrm{R}_{2}: \text { larger base radius. }
\end{aligned}
$$




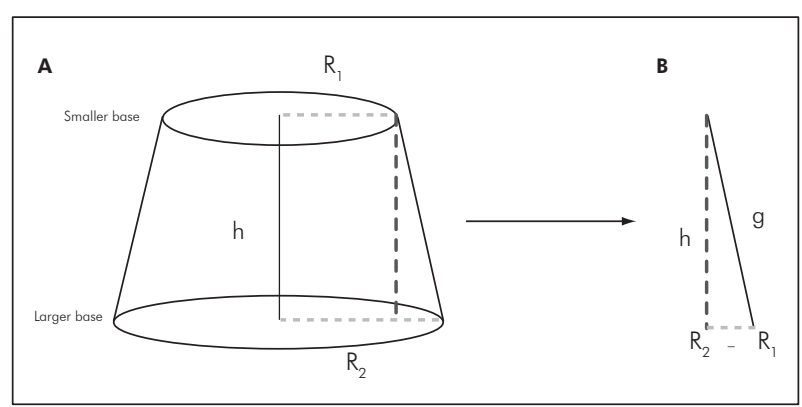

Figure 2 - (A) Schematic drawing corresponding to the internal section of the specimen (root walls: internal section of the specimen) - frustum of a right circular cone with parallel bases; (B) Geometric figure for calculating the generatrix of the cone frustum.

To determine the slant height the following calculation was used (Figure 2B):

$\mathrm{g}=\left(\mathrm{h}^{2}+\left[\mathrm{R}_{2}-\mathrm{R}_{1}\right]^{2}\right)^{1 / 2}$

where:

$\mathrm{H}$ : section height.

$\mathrm{R}_{1}$ and $\mathrm{R}_{2}$ were obtained by measuring the internal diameters of the smaller and larger base, respectively, which corresponded to the internal diameter of the root canal walls in the segment. These diameters were measured using a light microscope (Leica M-10 Wild, Gais, Switzerland), while h was measured with a digital caliper (Starrett ${ }^{\circledR} 727$, Starrett, Itu, Brazil).

The data obtained were submitted to analysis of variance (1-way ANOVA) and to the post hoc Tukey test $(\alpha=.05)$.

\section{Results}

The results of one-way ANOVA of the push-out bond strength data are shown in Table 1 . This analysis shows that the bond strength was affected by the mechanical cycling $(p=.0001)$.

The Tukey test showed that the mechanical cycling damaged the bonding of zirconia posts to root dentin significantly $(\mathrm{G} 1>\mathrm{G} 2=\mathrm{G} 3)$. The mean bond strength of G1 $(7.7 \pm 1.3 \mathrm{MPa})$ was statistically higher than those of G2 $(3.9 \pm 2.2 \mathrm{MPa})$ and G3 $(3.3 \pm 2.3 \mathrm{MPa})($ Graph 1$)$.

\section{Discussion}

Nowadays, a widely held concept is that fiber
Table 1 - ANOVA for the data (MPa) obtained in the pushout test

\begin{tabular}{l|c|c|c|c|c}
\hline \multicolumn{1}{c|}{ Effect } & df & SS & MS & $F$ & $p$-value \\
\hline Mechanical cycling & 2 & 114.304 & 57.1522 & 14.20 & $0.0001^{*}$ \\
\hline Residue & 27 & 108.554 & 4.0205 & & \\
\hline Total & 29 & 222.859 & & & \\
\hline${ }^{*} p<0.05$; df: degrees of freedom; SS: sum of squares; MS: mean square.
\end{tabular}

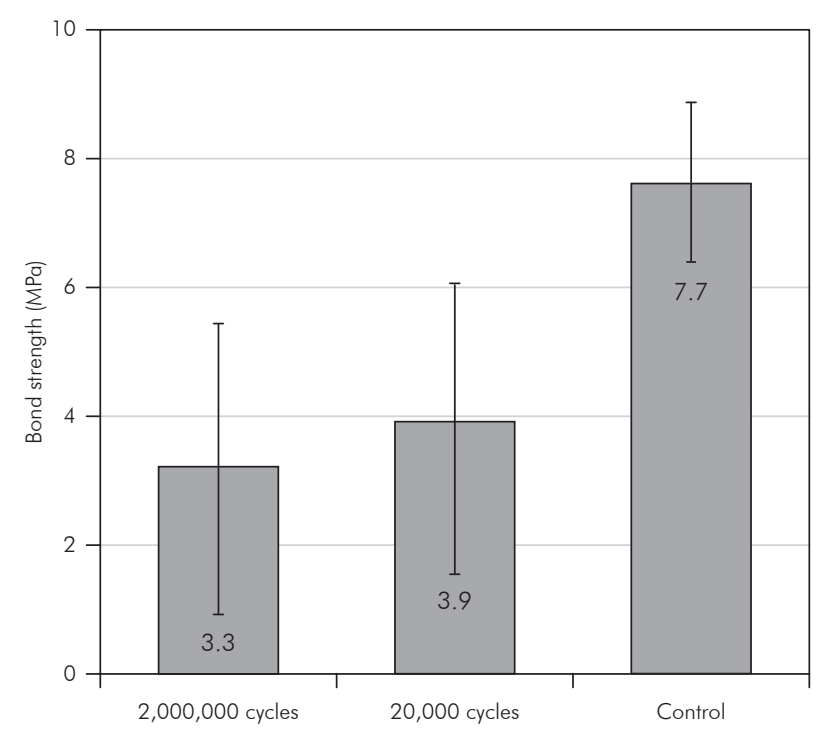

Graph 1 - Bond strength data (mean \pm standard deviation) $(\mathrm{MPa})$, according to the three different cycling conditions.

posts present mechanical advantages over ceramic posts because they have a modulus of elasticity $(\mathrm{E}=30)$ closer to that of dentin $(\mathrm{E}=18)$, permitting a better distribution of masticatory loads under clinical function. ${ }^{3,4,7,8,21,22}$ However, it is possible that this low modulus of elasticity causes bending of the fiber posts, generating stresses at the post/resin cement interface, damaging adhesion. , $^{5,7}$

Dietschi et al..$^{10}$ (1997) evaluated, under SEM, the effect of mechanical cycling on the bond strength between metallic posts and resin cement and between ceramic posts and resin cement. Significant debonding was observed when a ceramic post was used, which was not observed with metallic posts. According to the authors, although presenting a similar modulus of elasticity, the mechanism of adhesion between metallic post and resin cement is different from that between ceramic post and resin cement. With 
this in mind, the behavior of ceramic posts could not be based on the behavior of metallic posts. Similarly, in our study the bond strength of ceramic posts was decreased after mechanical cycling. This reduction might have occurred for the following reasons:

a. The combination of materials with different modulus of elasticity, since the ceramic posts present an "E" higher than those of dentin and resin cement. Thus, when a load is applied, it is transmitted to the softest material, the resin cement in this case.

b. Bond instability between post and cement during fatigue testing. The evaluation of the interface where failures occurred supports this statement. In the control group, $70 \%$ of the failures were predominantly between cement and dentin, whereas in Group 3, 75\% of the failures occurred predominantly between post and cement.

c. Crack propagation from bubbles and irregularities in the adhesive system or cement: The greater the number of irregularities, the smaller the bond strength of the set.

Such irregularities can occur as a result of the high C factor. Nikaido et al. ${ }^{23}$ (2002) evaluated the effects of mechanical cycling on the adhesive strength

\section{References}

1. Koutayas SO, Kern M. All-ceramic posts and cores: the state of the art. Quintessence Int. 1999;30(6):383-92. Review.

2. Mannocci F, Ferrari M, Watson TF. Intermittent loading of teeth restored using quartz fiber, carbon-quartz fiber, and zirconium dioxide ceramic root canal posts. J Adhes Dent. 1999;1(2):153-8.

3. Mannocci F, Pilecki P, Bertelli E, Watson TF. Density of dentinal tubules affects the tensile strength of root dentin. Dent Mater. 2004;20(3):293-6.

4. Pontius O, Hutter JW. Survival rate and fracture strength of incisors restored with different post and core systems and endodontically treated incisors without coronoradicular reinforcement. J Endod. 2002;28(10):710-5.

5. Qualtrough AJ, Mannocci F. Tooth-colored post systems: a review. Oper Dent. 2003;28(1):86-91. Review.

6. Heydecke G, Butz F, Hussein A, Strub JR. Fracture strength after dynamic loading of endodontically treated teeth restored with different post-and-core systems. J Prosthet Dent. $2002 ; 87(4): 438-45$. of resin materials by varying the C-factor. They noted a reduction in adhesive strength after fatigue test which was proportional to the $\mathrm{C}$-factor ratio. As the root canal provides the worst $\mathrm{C}$-factor, ${ }^{24}$ the presence of these irregularities might have contributed to the reduction in bond strengths after mechanical cycling.

Moreover, one important factor to be considered in endodontic restorations is the stress distribution when a mechanical load is applied. When a rigid endodontic post is used, there is a stress concentration at the apical portion. ${ }^{13,25}$ In this region, the dentin presents a great amount of inorganic material and, therefore, high fracture resistance. ${ }^{3}$ Thus, there is a compression of the adhesive/cement system that promotes the reduction in bond strength.

Even with the care taken in the present methodology, this study can only predict a reduction in bond strength performance, which should be confirmed by clinical longitudinal studies to assess the clinical performance of such adhesively cemented post.

\section{Conclusion}

Based upon the methodology applied, it can be concluded that the mechanical cycling affected the bond strength of a zirconia rigid post to root dentin.

7. Drummond JL, Toepke TR, King TJ. Thermal and cyclic loading of endodontic posts. Eur J Oral Sci. 1999;107(3):220-4.

8. Ottl P, Hahn L, Lauer HCH, Fay M. Fracture characteristics of carbon fibre, ceramic and non-palladium endodontic post systems at monotonously increasing loads. J Oral Rehabil. 2002;29(2):175-83.

9. Bolhuis HPB, de Gee AJ, Pallav P, Feilzer AJ. Influence of fatigue loading on the performance of adhesive and nonadhesive luting cements for cast post-and-core buildups in maxillary premolars. Int J Prosthodont. 2004;17(5):571-6.

10. Dietschi D, Romelli M, Goretti A. Adaptation of adhesive posts and cores to dentin after fatigue testing. Int J Prosthodont. 1997;10(6):498-507.

11. Huysmans MC, van der Varst PG, Schafer R, Peters MC, Plasschaert AJ, Soltesz U. Fatigue behavior of direct post-andcore-restored premolars. J Dent Res. 1992;71(5):1145-50.

12. Wiskott HW, Nicholls JI, Belser UC. Stress fatigue: basic principles and prosthodontic implications. Int J Prosthodont. 1995;8(2):105-16. Review. 
13. Cailleteau JG, Rieger MR, Akin JE. A comparison of intracanal stresses in a post-restored tooth utilizing the finite element method. J Endod. 1992;18(11):540-4.

14. Ari H, Yasar E, Belli S. Effects of $\mathrm{NaOCl}$ on bond strengths of resin cements to root canal dentin. J Endod. 2003;29(4):24851.

15. Baldissara P, Pieri F, Arcidiacomo A. Fatigue resistance of fiber posts: a comparison study [Abstract 1434]. J Dent Res. 2001;80:706.

16. Drummond JL, Bapna MS. Static and cyclic loading of fiberreinforced dental resin. Dent Mater. 2003;19(3):226-31.

17. Isidor F, Brondum K. Intermittent loading of teeth with tapered, individually cast or prefabricated, parallel-sided posts. Int J Prosthodont. 1992;5(3):257-61.

18. Kitasako Y, Burrow MF, Nikaido T, Harada N, Inokoshi S, Yamada $\mathrm{T}$ et al. Shear and tensile bond testing for resin cement evaluation. Dent Mater. 1995;11(5):298-304.

19. Van Noort R, Noroozi S, Howard IC, Cardew G. A critique of bond strength measurements. J Dent. 1989;17(2):61-7.
20. Pest L, Cavalli G, Bertani P, Gagliani M. Adhesive post-endodontic restorations with fiber posts: push-out tests and SEM observations. Dent Mater. 2002;18(8):596-602.

21. Akkayan B, Gülmez T. Resistance to fracture of endodontically treated teeth restored with different post systems. J Prosthet Dent. 2002;87(4):431-7.

22. Cormier CJ, Burns DR, Moon P. In vitro comparison of the fracture resistance and failure mode of fiber, ceramic, and conventional post systems at various stages of restoration. J Prosthodont. 2001;10(1):26-36.

23. Nikaido T, Kunzelmann KH, Chen H, Ogata M, Harada N, Yamaguchi $S$ et al. Evaluation of thermal cycling and mechanical loading on bond strength of a self-etching primer system to dentin. Dent Mater. 2002;18(3):269-75.

24. Bouillaguet S, Troesch S, Wataha JC, Krejci I, Meyer JM, Pashley DH. Microtensile bond strength between adhesive cements and root canal dentin. Dent Mater. 2003;19(3):199-205.

25. Ukon S, Moroi H, Okimoto K, Fujita M, Ishikawa M, Terada Y et al. Influence of different elastic moduli of dowel and core on stress distribution in root. Dent Mater J. 2000;19(1):50-64. 